\title{
Estudio teórico de las reacciones Diels-Alder utilizando plantillas de antraceno a través del uso de orbitales de frontera
}

\author{
A Diels-Alder Reaction Theoretical Study, by using Quiral Anthracene Groups, \\ Through the Frontier Orbitals Usage
}

R. Vivas-Reyes ${ }^{\mathrm{a}, *}$

K. M. Cardozo ${ }^{\mathrm{a}}$

J. F. Oviedo ${ }^{\mathrm{a}}$

Recepción: 27-mar-14

Aceptación: 07-ago-14

\begin{abstract}
Resumen
Se realizaron una serie de cálculos teóricos aplicados a una reacción de Diels-Alder, en los que se usaron una serie de plantillas de antraceno quiral y unos dienófilos derivados del anhidro maleico y maleimidas. Los cálculos fueron hechos con la ayuda del programa Gaussian 98, usando la funcional B3LYP junto con la base de cálculo 3-21G*. Con estos resultados fue posible analizar esta serie de compuestos, calculando su estabilidad energética y geometría, además de analizar el impacto que tienen sobre su reactividad los orbitales frontera HOMO y LUMO cuando los sustituyentes varían para una serie de plantillas y dienófilos. La energía de los orbitales frontera fue analizada y se pudo concluir que la reacción estudiada era del tipo de Diels-Alder de demanda normal de electrones y que los sustituyentes son importantes en el aumento de la reactividad de la reacción, encontrándose que el dieno, en este caso la plantilla de antraceno, hace aumentar la energía de los orbitales frontera cuando se encuentra unida a un sustituyente donante de electrones, y en cuanto a los dienófilos, se encontró que cuando se encuentran unidos a un sustituyente atractor de electrones, esto trae como consecuencia que se disminuya la energía de los orbitales frontera HOMO y LUMO. Respecto a la geometría de los orbitales frontera, se encontró que brinda información acerca de la estereoselectividad de la reacción, en donde el principio de conservación de la simetría orbital es el que rige la orientación de los sustituyentes en la reacción, en la cual se concluyó que para dienófilos sustituidos se observó que estos quedan orientados en la cara opuesta del centro estereogénico de la plantilla para este tipo de moléculas.
\end{abstract}

Palabras clave: DFT, HOMO, LUMO, Orbitales de frontera, Reacciones Diels-Alder.

a Departamento de Quimica. Grupo de Química Cuántica y Teórica de la Universidad de Cartagena, Campus De San Pablo Cartagena, Colombia.

*Autor de correspondencia: rvivasr@unicartagena.edu.co 


\begin{abstract}
A theoretical calculation' series was applied to a reaction of Diels-Alder, in which some series of quiral anthracene groups and dienofilos derived from the maleic anhydride and maleimides were used. The calculations were carried out in the program Gaussian 98 using the functional B3LYP along with the base $3-21 G^{*}$. The energy of the frontier orbitals was analyzed, and it was possible to conclude that the reaction was the type Diels-Alder of normal demand of electrons, which is useful to increase the reactivity when a diene is bonded to a substituent donator of electrons, because in this way the energy of orbitals HOMO-LUMO increases, and when the dienofiles were bonded to a substituent that attracts electrons, the energy of the orbitals HOMO-LUMO decreases. With respect to the orbital geometry of the frontier orbitals was found that stereo selectivity in the reaction was a guide under the conservation principle of the orbital symmetry.
\end{abstract}

Key words: DFT, Diels-Alder Reactions, Frontier Orbitals, HOMO, LUMO.

\section{Introducción}

La reacción de Diels-Alder es considerada como una de las reacciones pericíclicas más ampliamente estudiada en el campo de la síntesis orgánica [1,2]. Sus mecanismos, energías y algunas propiedades del estado de transición han sido extensamente estudiados en las pasadas décadas[3, 7]. Las cicloadiciones de Diels-Alder son procesos pericíclicos (redistribución cíclica de electrones de enlace) concertados, en los que se da la interacción entre un dieno (4 electrones $\pi$ ) y un dienófilo ( 2 electrones $\pi$ ) para formar, un anillo de seis miembros [6] En su forma más sencilla, la reacción de Diels-Alder combina butadieno con etileno para formar ciclohexano [9] tal como se muestra en la figura 1.

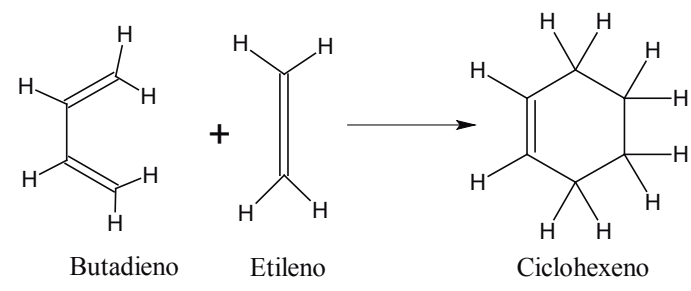

Figura 1. Reacción de butadieno y etileno para formar ciclohexano.

En el presente trabajo vamos a estudiar la reactividad y estéreoselectividad de la reacción de DielsAlder, para una serie de dienofilos y dienos que no han sido estudiados desde la perspectiva de los niveles de energía y geometría de los orbitales frontera. Es decir en términos del orbital de más alta energía ocupado HOMO (por sus siglas en inglés Highest Occupied Molecular Orbital) y el orbital de más baja energía desocupado LUMO (de sus siglas en inglés Lowest Unoccupied Molecular Orbital) [10].
Desde el punto de vista teórico existen varias interacciones posibles entre los orbitales de frontera del dieno y el dienófilo. En la figura 2(a) muestra que el HOMO del dieno reacciona con el orbital LUMO de baja energía del dienofilo. Es llamada en una reacción de Diels-Alder llamada de demanda normal de electrón, en la cual la diferencia de energía entre el HOMO del dieno y el LUMO del dienófilo gobierna la velocidad de la reacción. El anhidro maleico es un dienófilo representativo de este tipo de comportamiento [6].

En la figura 2(b) se observa la interacción la cual el dieno reacciona con un dienófilo tal como etileno, que no posee un LUMO bajo ni un HOMO alto. Este tipo de reacciones posee una baja reactividad y necesita condiciones de reacción relativamente más severas para producir la reacción de Diels-Alder [10], debido a la mayor diferencia de energía entre los orbitales involucrados.

La figura 2(c) muestra la combinación en la cual el dieno reacciona con un dienófilo que posee un nivel energético HOMO más alto que el HOMO del dieno. Este tipo de reacción se conoce como reacción de Diels-Alder de demanda inversa de electrón, en la cual la diferencia de energía entre el orbital HOMO del dienófilo y el LUMO del dieno gobiernan la velocidad de la reacción. Sin embargo, teniendo en cuenta que generalmente es el dieno la especie que aporta los electrones y el dienófilo es el que los recibe, este tipo de reacción rara vez ocurre en comparación con la reacción de Diels-Alder de demanda normal de electrón y son ejemplo de éstas, en las que participan dienófilos con grupos electrón-dadores como los éteres vinílicos. 
Estudio teórico de las reacciones Diels-Alder utilizando plantillas de antraceno a través del uso de orbitales de frontera

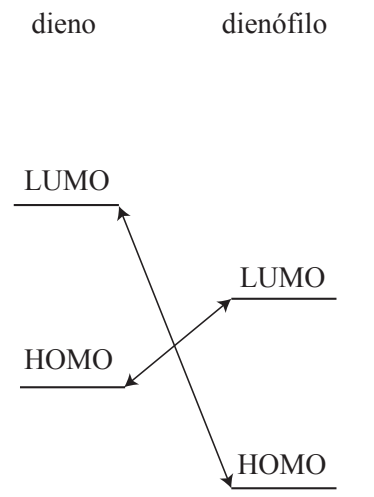

a) Demanda normal de electrones

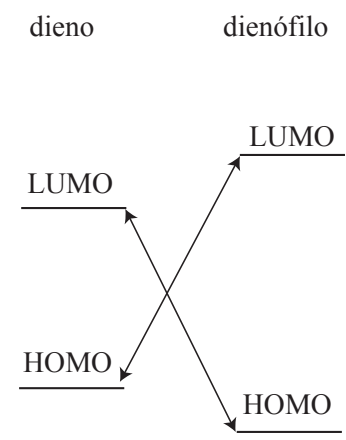

b)

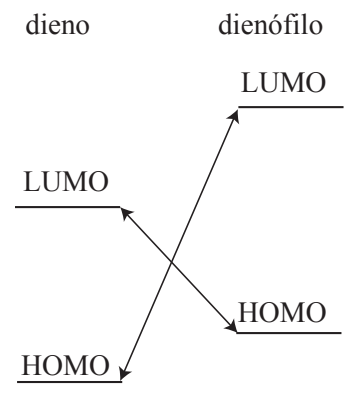

c) Demanda inversa de electrones

Figura 2. Tipos de interacción entre los orbitales de frontera con base a la relación de niveles de energía entre HOMO-LUMO de dieno y dienófilo, para la reacción de Diels-Alder.

A través de los cálculos teóricos hechos sobre los diferentes dienos y dienofilos objeto de este trabajo, se pudo mostrar que la energía y geometría de los orbitales moleculares de frontera HOMO-LUMO son descriptores confiables para representar la reactividad y estéreo-selectividad de las reacciones DielsAlder estudiadas y sus análogas, además de mostrar que los valores energéticos de los orbitales de frontera se ven afectados por el carácter del sustitúyete tanto del dieno como del dienofilo, como ya ha sido planteado previamente desde el análisis de demanda electrónica [11].

\subsection{Teoría y métodos computacionales}

La Teoría de los Orbitales de Frontera considera a los electrones del HOMO como los electrones de valencia y por lo tanto supone que son los que más probablemente controlarán el modo de reacción del sistema. Así es como la teoría considera que la interacción entre un sustrato y un reactivo tiene lugar mediante solapamiento de orbitales vacíos del sustrato y orbitales llenos del reactivo y viceversa. Aunque todos los O.M. interaccionan, la intensidad es mayor cuanto más próximos en energía estén, así las interacciones más intensas se espera sean producto de la aproximación entre orbitales frontera. Si esas interacciones intensas pueden darse, la reacción diremos que está permitida y se realizará fácilmente vía Concertada [10]. El análisis de la aproximación de estos orbitales de frontera, teniendo en cuenta la teoría de conservación de la simetría orbital, permite obtener información muy valiosa acerca de la estéreoselectividad de la reacción, como ha sido descrito por ejemplo, en el estudio teórico a nivel $a b$ initio de reactividad y estereoselectividad para la reacción de heterodienofilos con el furano [11] y del 2-azabutadieno con aldehídos [12].

Las moléculas utilizadas en los cálculos en este estudio fueron tomadas del trabajo de Snyder y colaboradores [12, 13], en los que se resalta la alta estereoselectividad que presentan las cicloadiciones de antracenos quirales con diversos dienofilos, y la influencia del grupo trifluorometil en posición 9 (ver figura 3). Las estructuras tanto de las plantillas de antracenos, como de los dienofilos (anhidro maleico, maleimidas y derivados, ver figura 4 ), se optimizaron, usando el conjunto de programas Gaussian [14] con el método AM1 [15] y luego los resultados obtenidos se usaron como punto de partida para posteriormente ser tratados a un nivel de cálculo mas alto como fue el funcional B3LYP [16] junto con la base de cálculo 3-21G* [17].

Una vez optimizadas la geometría de las moléculas, tanto de plantillas como dienofilos, se calcularon los valores energéticos de los orbitales de frontera HOMO-LUMO y las cargas netas sobre los átomos involucrados en el enlace. Estos cálculos se realizaron utilizando el funcional B3LYP y la base de cálculo 3-21G*.

Los valores numéricos calculados de los orbitales moleculares HOMO y LUMO, tanto de los dienófilos del I al XI (figura 4) como de las plantillas 1 a 5, son mostrados en la tabla 1.

El valor numérico obtenido para el orbital molecular HOMO (ver tabla 1) muestra que las plantillas 2 y 4 tienen los valores energéticos para el orbital HOMO más altos más bajos de la serie, $-5,18$ eV y -5,06 


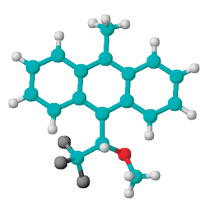

Plantilla 1

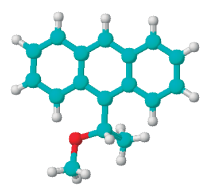

Plantilla 2

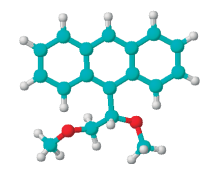

Plantilla 4

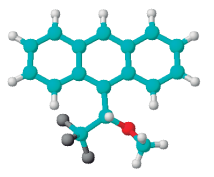

Plantilla 3

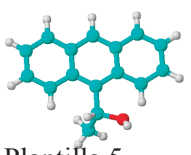

Plantilla 5

Figura 3. Estructuras de plantillas de antraceno. Plantilla 1=9-(1-metoxi-2.2.2 trifluoroetil)-10-metil-antraceno, Plantilla 2 = 9-(1-metoxietil) antraceno, Plantilla 3 = 9-(1- metoxi-2, 2,2-trifluoroetil) antraceno, Estructuras de plantillas de antraceno. Plantilla 1= 9-(1-metoxi-2.2.2 trifluoroetil)-10-metil-antraceno, Plantilla $2=9$-(1-metoxietil) antraceno, Plantilla $3=9-(1-$ metoxi-2, 2,2-trifluoroetil) antraceno, Plantilla $4=9$-(1,2-dimetoxietil) antraceno, Plantilla* $=9$-(1- hidroxietil) antraceno. $=$ 9-(1,2-dimetoxietil) antraceno, Plantilla* $=9$-(1- hidroxietil) antraceno.

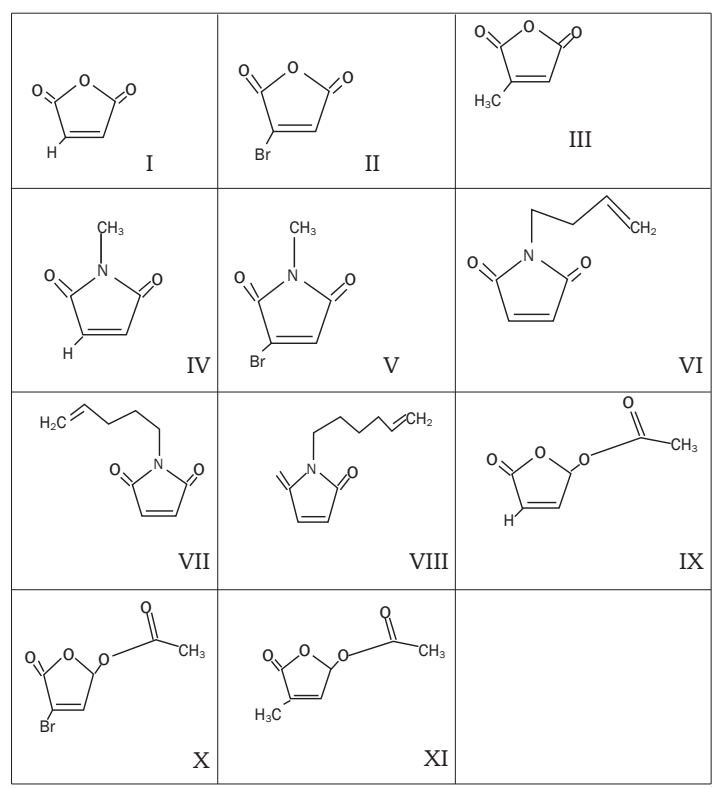

Figura 4. Estructura de los dienófilos, en donde: I) anhidro maleico, II) anhidro bromo maleico, III) anhidro 2-metil maleico, IV) N-metil maleimida, V) 2-bromo $\mathrm{N}$-metil maleimidas, VI) N-butenil maleimida, VII) $\mathrm{N}$ pentenil maleimida, VIII) $\mathrm{N}$-hexenil maleimida, IX) 2-acetil-ciclopenten lactona, X) 2-acetil-4-bromo ciclopenten lactona y XI) 2-acetil-4 metil ciclopenten lactona.

$\mathrm{eV}$, respectivamente, lo cual hace que estas plantillas tengan más afinidad para el tipo de dienófilos que las otras, ya que de la diferencia de energía que exista entre el orbital HOMO del dieno y el orbital LUMO del dienófilo es de gran importancia para este tipo de reacciones, como descriptor molecular, debido a que nos brinda gran información acerca de la reactividad. Continuando con el análisis de estas dos plantillas, con el propósito de establecer correlaciones con los resultados experimentales reportados previamente
Tabla 1. valores de energía (EV) de los orbitales HOMO-LUMO para los dienófilos y plantillas de dienos, corridas a un nivel de cálculo B3LYP/ 3-21G*.

\begin{tabular}{lcc}
\hline estructuras & HOMO $(\mathrm{eV})$ & LUMO $(\mathrm{eV})$ \\
\hline dienófilo 1 & $-8,00$ & $-3,24$ \\
dienófilo 2 & $-8,19$ & $-3,37$ \\
dienófilo 3 & $-7,83$ & $-2,94$ \\
dienófilo 4 & $-7,20$ & $-2,57$ \\
dienófilo 5 & $-7,35$ & $-2,74$ \\
dienófilo 6 & $-7,00$ & $-2,62$ \\
dienófilo 7 & $-6,87$ & $-2,63$ \\
dienófilo 8 & $-6,89$ & $-2,57$ \\
dienófilo 9 & $-7,50$ & $-2,00$ \\
dienófilo 10 & $-7,53$ & $-2,17$ \\
dienófilo 11 & $-7,38$ & $-1,73$ \\
plantilla 1 & $-5,29$ & $-1,77$ \\
plantilla 2 & $-5,18$ & $-1,57$ \\
plantilla 3 & $-5,40$ & $-1,81$ \\
plantilla 4 & $-5,06$ & $-1,46$ \\
plantilla 5 & $-5,19$ & $-1,59$ \\
\hline
\end{tabular}

$[11,12,18,19,20]$, en los que se señala claramente que la naturaleza de los sustituyentes influencia la energía de los orbitales, cambiando su separación. En el caso de los sustituyentes electro-atrayentes, la separación energética HOMO-LUMO se disminuye, dando como resultado que el estado de transición se estabilice [11].

La comparación entre las diferencias que existen entre los orbitales HOMO-LUMO del dienodienófilo y el HOMO-LUMO y del dienófilo-dieno, para el antraceno representado en la plantilla 2 y los anhídridos maleico (dienófilo 1) y bromomaleico (dienófilo 2), se hace con el fin de establecer qué tipo de interacción se puede dar entre los orbitales frontera [10], tal como se muestra en las figuras $5 \mathrm{y}$ 6. 


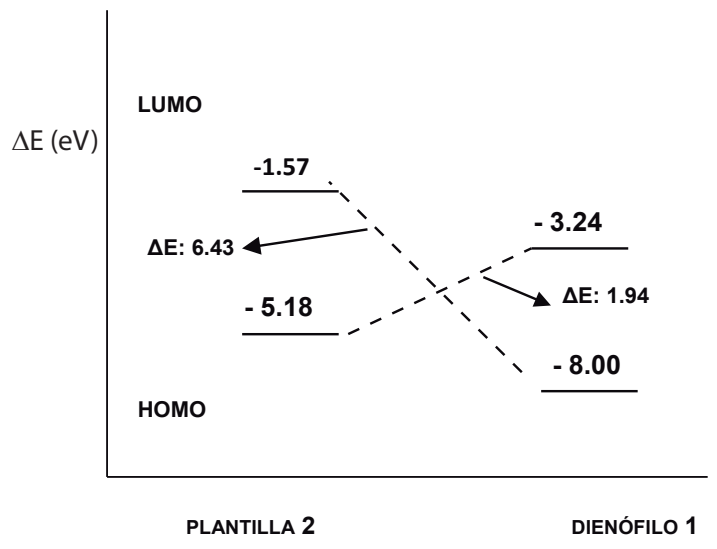

Figura 5. Diagrama de energía (eV) de los orbitales frontera para plantilla 2 con el dienófilo 1 , obtenido a un nivel de cálculo B3LYP/3-21G*.

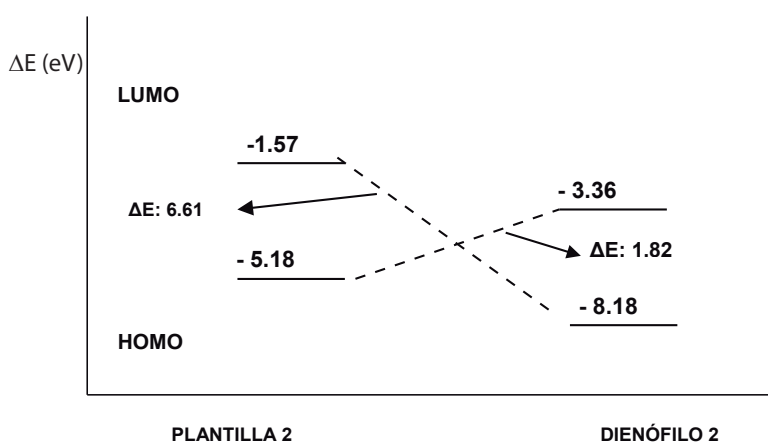

Figura 6. Diagrama de energía (eV) de los orbitales frontera para la plantilla 2 con el dienófilo 2, obtenido a un nivel de cálculo B3LYP/3-21G*.

Si se observan las diferencias energéticas $(\Delta \mathrm{E})$, se ve claramente que la menor diferencia se da cuando la interacción es entre el orbital HOMO del dieno con el orbital LUMO de ambos dienófilos, de lo cual se puede concluir que el tipo de reacción Diels-Alder es una del tipo de demanda normal de electrones, en la cual el dieno es una molécula rica en electrones, y el dienófilo es deficiente en electrones. Adicionalmente, como la diferencia de energía entre los orbitales HOMO dieno-LUMO dienófilo vs. HOMO dienófilo-LUMO dieno de las demás estructuras permite observar la misma tendencia, se puede decir que la reacción que se da entre las diferentes plantillas y dienófilos procede de la misma forma, es decir, por una reacción Diels-Alder de demanda normal de electrones.

Ahora, analizando las estructuras de las plantillas 2 y 4 (ver figura 3 ), se observa que en ambos casos el sustituyente en el carbono 9 del antraceno tiene un carácter donante de electrones, debido a la presencia del grupo metoxi, lo cual favorece la reacción Diels-
Alder de demanda normal de electrones, porque al aumentar la densidad electrónica del dieno, aumenta la energía del HOMO. Se ve que para la plantilla 2 el valor del orbital HOMO es $-5,18 \mathrm{eV}$, y que en esta estructura hay un sustituyente etilo sobre el átomo de Carbono 9 del antraceno con un grupo metoxi en posición 1', en comparación con la plantilla 4, cuyo valor de energía del orbital HOMO es más alto, de $-5,06 \mathrm{eV}$, lo cual se justifica debido a que se observa que tiene dos grupos metoxi en posiciones 1' y 2', lo que ocasiona que aumente aún más la densidad electrónica de la molécula, haciéndola más reactiva [22] ante dienófilos que tengan un LUMO de baja energía, como los presentes en este estudio.

El caso de las plantillas 1 y 3 , que tienen una molécula atractora de electrones, como es el grupo trifluoro etil en posición 9 al antraceno, presenta, según reflejan los cálculos, una disminución de la densidad electrónica, comprometiendo su reactividad en una reacción Diels-Alder, lo cual concuerda con lo que se ha encontrado desde el punto de vista experimental [20-22]. Para el caso específico de la plantilla 1, este efecto no es tan marcado, pues también cuenta con la presencia del grupo electrón dador en posición 10, que se presume alcanza a compensar en algo la pérdida de la densidad de carga originada por el trifluorometil en posición 1' sobre el metil en posición 9. El hidroxi es común en ambas estructuras, y se supone afectará la reactividad de ambas plantillas de forma similar.

En el caso de los dienófilos (ver figura 4) se observa cómo es afectada la energía de los orbitales HOMO-LUMO de las estructuras con los diferentes sustituyentes; comenzamos por analizar el caso del dienófilo 1 (ver figura 5), el anhidro maleico, el cual tiene un valor de energía para el orbital molecular HOMO de $-8,00 \mathrm{eV}$, mientras que para el dienófilo 2 el valor de energía para el orbital HOMO es de $-8,18 \mathrm{eV}$; el dienófilo 2 tiene un átomo de bromo en posición 3 , el cual por su carácter atractor de electrones hace que se disminuya la densidad electrónica del doble enlace, convirtiendo la molécula en una especie con mayor afinidad frente a los dienófilos, lo cual se refleja a través de los cálculos, debido a que la diferencia energética entre los orbitales frontera HOMO del dieno y LUMO del dienófilo, de $1,82 \mathrm{eV}$, presenta una leve reducción con respecto al anhídrido maleico (dienolfilo 1), (ver figura 5). 
Tabla 2. Diferencia energética $(\mathrm{eV})$ de los orbitales frontera de la plantilla $2(5,18 \mathrm{eV})$ con cada uno de los dienófilos.

\begin{tabular}{lcl}
\hline Estructura & LUMO $(\mathrm{eV})$ & $\begin{array}{l}\Delta \text { HOMO-LUMO } \\
\text { plantilla-dienófilo }(\mathrm{eV})\end{array}$ \\
\hline dienófilo 1 & $-3,24$ & 1,94 \\
dienófilo 2 & $-3,37$ & 1,82 \\
dienófilo 3 & $-2,94$ & 2,24 \\
dienófilo 4 & $-2,57$ & 2,61 \\
dienófilo 5 & $-2,74$ & 2,44 \\
dienófilo 6 & $-2,62$ & 2,56 \\
dienófilo 7 & $-2,63$ & 2,55 \\
dienófilo 8 & $-2,57$ & 2,61 \\
dienófilo 9 & $-2,00$ & 3,18 \\
dienófilo 10 & $-2,17$ & 3,01 \\
dienófilo 11 & $-1,73$ & 3,45 \\
\hline
\end{tabular}

De la tabla 2 se puede decir que para el caso entre la plantilla 2 con el dienófilo 3 , un dienófilo que tiene un sustituyente metilo en posición 3 , donante de electrones, este hace aumentar la densidad electrónica del dienófilo, teniendo como efecto un aumento de la energía del orbital HOMO de la molécula, cuyo valor es de $-7,83 \mathrm{eV}$, que es más bajo que el valor mostrado por los dienófilos 1 y 2 , ocasionando que la diferencia energética entre el orbital HOMO del dieno y el orbital LUMO [10] del dienófilo aumente, lo que termina en una disminución de la reactividad de la reacción entre estas 2 moléculas, ya que esta va a depender de este valor que, para este caso, es de $2,24 \mathrm{eV}$.

La diferencia energética (ver tabla 2) entre la plantilla 2 con el dienófilo 4, la N-maleimida, cuyo nitrógeno está sustituido por un grupo metilo, que por su carácter donante de electrones y también por la alta electronegatividad del nitrógeno. El heteroátomo del anillo ahora es el nitrógeno, menos electronegativo que el oxígeno, y en consecuencia permite una mayor deslocalización con los carbonilos, lo que entra en competencia con la deslocalización de los electrones $\pi$ del doble enlace; esto, adicional a la presencia del grupo metilo sobre el heteroátomo, como electrón dador, hace que se incremente la densidad electrónica de los orbitales moleculares HOMO y LUMO, comparativamente con la observada del anhídrido maléico, y esto lleva a un aumento en el $\Delta \mathrm{E}$. El nivel de cálculo utilizado alcanza a reflejar este cambio en la reactividad de $2,61 \mathrm{eV}$ vs. 1,94 del maleico; esto lleva a una disminución de la reactividad, concordando así con lo encontrado experimentalmente $[20,21]$.
Caso parecido sucede entre la reacción de la plantilla 2 con el dienófilo 5 (ver tabla 2), en donde ahora el efecto discutido anteriormente se ve a perturbado por un grupo atractor de electrones unido al carbono número 3 del doble enlace, como lo es el bromo, cuya consecuencia es que disminuye un poco la densidad electrónica que había ganado la estructura y que la diferencia energética sea un poco más baja, aumentando así la reactividad de esta reacción en comparación con el caso anterior. En este caso, el dienófilo 6 (ver tabla 2) tiene una cadena alifática, que aumenta la densidad electrónica del anillo debido a la atracción del átomo de nitrógeno por tener una electronegatividad alta, atrayendo la densidad electrónica presente en la cadena alifática hasta el anillo, disminuyendo así su reactividad en la reacción Diels-Alder de este dienófilo con la plantilla.

Ahora se observa cómo la reactividad de la reacción del dienófilo 8 (ver tabla 2) disminuye debido a que aún se sigue dando el proceso de transferencia electrónica de la cadena alifática hacia el anillo, disminuyendo un poco la densidad electrónica sobre el anillo; esto se debe a que el sistema pi del doble enlace ubicado en el extremo de la cadena se encuentra ahora más distanciado y su aporte de electrones ha disminuido por encontrarse más lejos, y se puede decir que este efecto tiene una limitación cuando la cadena alifática se va alargando, llegando un momento en que su efecto sobre el anillo no es relevante cuando aumenta el número de carbonos en la cadena alifática y se aleja el doble enlace del anillo.

Para el caso de la reacción de la plantilla con el dienófilo 9 (ver tabla 2), que tiene un grupo acetato como sustituyente en el anillo, que aunque no está directamente enlazado sí contribuye con el aumento de la densidad electrónica, debido a que es un donante de electrones y, por lo tanto, se vuelve menos reactivo en una reacción Diels-Alder, lo cual también se ve reflejado en la diferencia energética entre los orbitales HOMO-LUMO de la plantilla y el dienófilo, obteniéndose un valor de $3,18 \mathrm{eV}$.

Los valores de los orbitales HOMO-LUMO entre el dienófilo 10 con la plantilla 2 (ver tabla 2) indican una diferencia entre la energía de los orbitales frontera de 3,02 eV; observando la estructura del dienófilo se aprecia que es parecida a la del dienófilo 9 , que tiene un aporte electrónico del grupo acetato al anillo y, además, un grupo atractor de electrones en 
uno de los carbonos involucrados en el doble enlace, como es el caso del átomo de bromo, que disminuye la densidad de carga, viéndose esto reflejado en un pequeño aumento de la reactividad de la reacción en comparación con la anterior reacción.

Para el dienófilo 11, la diferencia energética entre los orbitales HOMO-LUMO es la más alta de la serie, con un valor 3,45 eV (ver tabla 2), debido a que si se analiza la estructura del dienófilo (ver figura 4) se ve que hay dos grupos donadores de electrones unidos al anillo, como son el grupo acetato y el grupo metilo que hacen aumentar la densidad electrónica de este dienófilo, aumentando así tanto la energía del orbital HOMO como la del orbital LUMO, y, por lo tanto, la reactividad de la reacción Diels-Alder de la plantilla 2 con este dienófilo va a ser baja.

Usando de nuevo los valores mostrados en la tabla 2, y para resumir los resultados antes obtenidos, se puede observar que las diferencias energéticas que existen para los orbitales HOMO y LUMO de la platilla 2 con los diferentes dienófilos, en la cual los valores más bajos corresponden a las reacciones que teóricamente pueden proceder con una alta reactividad, y los valores altos deberían corresponder a reacciones entre reactivos con poca probabilidad de reaccionar entre sí. El orden de reactividad para las diferentes reacciones es:

plantilla2-dienófilo2 > plantilla2-dienófilo1 > plantilla2-dienófilo3 > plantilla2-dienófilo5 > plantilla2-dienófilo7 > plantilla2-dienófilo6 > plantilla2-dienófilo8 > plantilla2-dienófilo4 > plantilla2-dienófilo10 > plantilla2-dienófilo9 > plantilla2-dienófilo11

En la tabla 3 se presentan los resultados del comportamiento de cada uno de los dienófilos con la plantilla 4, tal como se hizo para el caso de la planti1la2. Se observa que el $\Delta \mathrm{E}$ entre los orbitales frontera de la plantilla 4 (que tiene los orbitales HOMO y LUMO con más alta energía que los planteados en la plantilla 2) y los orbitales de los dienófilos.

En la figura 7 se observa cómo la diferencia energética entre los orbitales moleculares HOMOLUMO es menor cuando interacciona el orbital HOMO de la plantilla 4 y orbital LUMO del dienófilo 1, lo que indica que esta reacción Diels-Alder es de demanda normal de electrones, como pasó en el caso analizado anteriormente, en donde el dieno es rico en electrones y el dienófilo es pobre en estos.

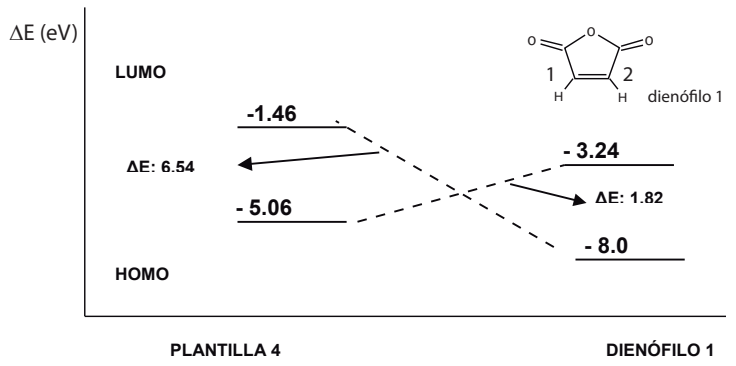

Figura 7. Diagrama de energía (eV) de los orbitales frontera para la plantilla 4 con el dienófilo 1 , obtenido a un nivel de cálculo B3LYP/3-21G*.

Ahora, la diferencia energética entre estos orbitales de frontera, de $1,82 \mathrm{eV}$, es inferior a la presentada en la plantilla 2 con el mismo dienófilo; posiblemente, debido a la presencia de dos sustituyentes metoxi electrono-donadores sobre el etilo en posición 9 en la plantilla 4, en comparación con un solo metoxi en la plantilla 2. Lo anterior origina mayor deficiencia de carga y, en consecuencia, disminución de la energía de sus orbitales de frontera.

En el caso de la reacción de la plantilla 4 con el dienófilo 3 (ver tabla 3 ) hay un sustituyente donante de electrones, como es el grupo metilo, que afecta la densidad electrónica de la molécula, ocasionando que el valor energético de los orbitales frontera de la molécula aumente, mostrando un valor de 7,83 eV para el HOMO y de 2,94 eV para el LUMO, y se vea así comprometida la reactividad de la reacción, ya que la diferencia energética entre los orbitales frontera aumentará a un valor de $2,12 \mathrm{eV}$ y, como se mencionó anteriormente, a medida que aumente este valor la reactividad decrece.

Tabla 3. Diferencia energética (eV) entre los orbitales frontera de la plantilla $4(5,06 \mathrm{eV})$ con cada de uno de los dienófilos.

\begin{tabular}{lll}
\hline Estructura & $\begin{array}{l}\text { LUMO }(\mathrm{eV}) \\
\text { dienófilo }\end{array}$ & $\begin{array}{l}\Delta \text { HOMO-LUMO } \\
\text { plantilla-dienófilo } \\
(\mathrm{eV})\end{array}$ \\
\hline dienófilo 1 & $-3,24$ & 1,82 \\
dienófilo 2 & $-3,37$ & 1,67 \\
dienófilo 3 & $-2,94$ & 2,12 \\
dienófilo 4 & $-2,57$ & 2,49 \\
dienófilo 5 & $-2,74$ & 2,33 \\
dienófilo 6 & $-2,62$ & 2,45 \\
dienófilo 7 & $-2,63$ & 2,44 \\
dienófilo 8 & $-2,57$ & 2,49 \\
dienófilo 9 & $-2,00$ & 3,06 \\
dienófilo 10 & $-2,17$ & 2,89 \\
dienófilo 11 & $-1,73$ & 3,33 \\
\hline
\end{tabular}


Se observa que la diferencia energética entre los orbitales moleculares HOMO-LUMO es menor cuando interaccionan el orbital HOMO de la plantilla 4 y el orbital LUMO del dienófilo 4 (ver tabla 3), lo que nos dice que la reacción Diels-Alder es también una reacción del tipo de demanda normal de electrones, en donde el dieno es rico en electrones, y el dienófilo es pobre en estos.

Ahora, mirando la diferencia energética de los orbitales frontera se tiene que es igual a $2,49 \mathrm{eV}$; esta diferencia es menor para la plantilla 2 con este mismo dienófilo, lo cual se debe a que el valor de los orbitales frontera de la plantilla 4 es mayor porque los sustituyentes en el carbono quiral son de carácter donante de electrones, como son los dos grupos metoxi unidos a él; en el caso de la plantilla 2, está también tiene dos grupos donantes, como lo son el grupo metoxi y el metilo, pero como el grupo metilo tiene un carácter donante de electrones menor que el grupo metoxi ocasiona que tenga valores menores de los orbitales HOMO-LUMO.

Caso parecido sucede en la reacción de la plantilla 4 con el dienófilo 5 (ver tabla 3), en donde el efecto discutido anteriormente se ve perturbado por un grupo atractor de electrones unido al carbono número 1 del doble enlace, como lo es el bromo, cuya consecuencia es la disminución de la densidad electrónica que había ganado la estructura debido a la atracción que ejercía el átomo de nitrógeno sobre un grupo donante, como lo es el grupo metilo, ocasionando que la diferencia energética sea un poco más baja y aumentando así la reactividad de esta reacción en comparación con la del caso anterior.

En el caso de la plantilla 4 con el dienófilo 6 (ver tabla 3), este tiene una cadena alifática que hace aumentar la densidad electrónica del anillo debido a la atracción del átomo de nitrógeno, que tiene una electronegatividad más alta, atrayendo la densidad electrónica presente en la cadena alifática hasta el anillo, y de esta forma hace que se disminuya la reactividad de la reacción Diels-Alder del tipo de demanda normal de electrones en la cual el dienófilo debe tener una baja densidad electrónica para así favorecer la reacción [22], pero en este caso el dienófilo está influenciado por la cadena alifática que se encuentra unida al átomo de nitrógeno.

El dienófilo 7 (ver tabla 3) tiene las mismas características que el dienófilo 6, a diferencia de que la cadena alifática tiene un átomo de carbono más, lo cual le confiere un poco más de densidad electrónica al anillo, lo que repercute en un valor un poco mayor que para el caso anterior, esperándose que la reacción entre este dienófilo y la plantilla 4 sea menor, comparada con el caso anterior.

Para la reacción entre el dienófilo 8 y la plantilla 4 (ver tabla 3 ), se observa cómo la reactividad de la reacción del dienófilo disminuye debido a que aún se sigue dando el proceso de transferencia electrónica de la cadena alifática hacia el anillo, esto se debe a que el sistema pi del doble enlace, ubicado en el extremo de la cadena, se encuentra ahora más distanciado y su aporte de electrones al anillo disminuye, y, como se dijo antes, este efecto tiene una limitación cuando la cadena alifática se va alargando, llegando un momento en que su efecto sobre el anillo no es relevante y no afecta en gran medida el valor energético de los orbitales frontera.

Para el caso de la reacción de la plantilla 4 con el dienófilo 9 (ver tabla 3), este tiene un grupo acetato como sustituyente en el anillo, el cual, aunque no está directamente enlazado, sí contribuye con el aumento de la densidad electrónica debido a que es un donante de electrones y, por lo tanto, se vuelve menos reactivo en una reacción Diels-Alder, lo cual también se ve reflejado en la diferencia energética entre los orbitales HOMO-LUMO de la plantilla y el dienófilo, obteniéndose un valor de 3,06 eV.

La diferencia entre los orbitales moleculares HOMO-LUMO del dienófilo 10 con la plantilla 4 es de $2.89 \mathrm{eV}$; observando la estructura del dienófilo se observa que es parecida a la del dienófilo 9; esta estructura tiene un aporte electrónico del grupo acetato al anillo y, además, un grupo atractor de electrones en uno de los carbonos involucrado en el doble enlace, como es el caso del átomo de bromo, que disminuye la densidad de carga, teniendo como consecuencia un pequeño aumento de la reactividad de la reacción en comparación con el caso anterior.

Para el dienófilo 11 la diferencia energética entre los orbitales HOMO-LUMO (ver tabla 3 ) es la más alta de la serie, con un valor $3,33 \mathrm{eV}$, debido a que si analizamos la estructura del dienófilo se ve que los dos grupos donadores de electrones unidos al ani1lo, como el grupo acetato y el grupo metilo, hacen aumentar la densidad electrónica de este dienófilo, aumentando así tanto la energía del orbital HOMO 
como la del orbital LUMO y, por lo tanto, la reactividad de la reacción Diels-Alder de la plantilla 4 con este dienófilo va a ser baja.

Durante muchos años no se comprendieron bien las reacciones pericíclicas y se consideraban impredecibles; para predecir los resultados se consideró la simetría de los orbitales moleculares de los reactivos y productos dentro de la llamada teoría conservación de la simetría orbital, la cual establece que los orbitales moleculares de los reactivos deben calzar o coincidir con los orbitales moleculares de los productos sin cambios drásticos en la simetría [22]. Ahora, según los resultados obtenidos para las diferentes moléculas (figura 8) tenemos que:

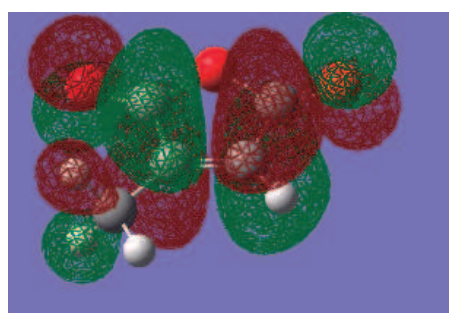

a)

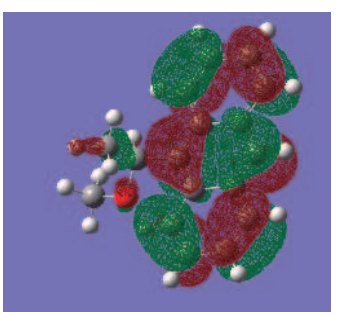

b)
Figura 8. Diagrama de energía (eV) de los orbitales frontera para la plantilla 4 con el dienófilo 1 , obtenido a un nivel de cálculo B3LYP/3-21G*.

\section{Conclusiones}

Para la reacción de Diels-Alder del tipo estudiada en este trabajo es importante contar, a nivel teórico, con parámetros que permitan inferir sobre la estabilidad de los sistemas de dienos y dienófilos.

La energía y la geometría de los orbitales moleculares frontera resultaron ser un descriptor muy significativo y confiable para describir la reactividad y estereoselectividad de una reacción como la de Diels-Alder, ya que su comportamiento está en gran medida gobernado por las energías de los orbitales HOMO-LUMO y por las geometrías de estos, las cuales mostraron una gran variabilidad con los sustituyentes involucrados en los sistemas moleculares que tomaban parte de esta reacción.

Luego del análisis de los orbitales frontera, se observó cómo los valores de las energías se ven afectados por el carácter del sustituyente, ya sea este donante o atractor de electrones, y si a esto le añadimos el tipo de interacción de los orbitales frontera, que nos indicará el tipo de reacción Diels-Alder que se va a llevar a cabo, que en este estudio resultó ser una reacción de demanda normal de electrones, en la cual el dienófilo es pobre en electrones y el dieno es rico en estos y, para este caso, tiene un grupo donante de electrones, este ocasiona que se vea aumentada la densidad electrónica, y esto se ve reflejado en el hecho de que exista un incremento de la energía de los orbitales moleculares HOMO y LUMO de la molécula; para el caso contrario, el dienófilo con un sustituyente atractor de electrones hace disminuir la densidad electrónica y, por lo tanto, ocasiona que disminuya la diferencia energética entre los orbitales moleculares HOMO-LUMO, y dieno y dienófilos con estas características harán que la reactividad de la reacción se vea aumentada considerablemente.

En cuanto a las plantillas, se vio que las menores diferencias energéticas fueron observadas en la plantilla 4 con los dienófilos del estudio, mostrando que puede existir una buena reactividad entre estos reactivos, lo cual concuerda perfectamente con los hechos experimentales para la plantilla 4. La plantilla 2 también mostró una diferencia energética baja, pero no inferior a la mostrada por la plantilla 4 , evidenciando que tiene una buena reactividad con el tipo de dienófilos estudiados aquí, lo cual también concuerda con lo realizado a nivel experimental; además, la geometría de los orbitales frontera, si se tiene en cuenta el principio de simetría orbital, mostró que la estereoselectividad puede ser explicada muy bien, en donde se encontró que para dienófilos sustituidos el sustituyente queda orientado en la cara puesta al centro estereogénico de la plantilla.

\section{Agradecimientos}

Los autores agradecen a la Universidad de Cartagena por su permanente apoyo a la realización de esta investigación; adicionalmente, a un árbitro anónimo que con sus sugerencias mejoró notablemente la calidad de este artículo.

\section{Referencias}

[1] H. Wei-Ping, H. Chun-Huei y T. Li-Chao, "Dual-Level Direct Dynamics Study on the Diels -Alder Reaction of Ethylene and 1,3Butadiene". J. Phys. Chem. A, 105: 9945-9953, 2001. 
[2] O. Yasuharu, O. "Large Enhacement of Reactivity of Diels -Alder Reactions on a C(001)(2x1) Surface: A Hibrid Density- Functional Study". J. Phys. Chem. B, 105: 1813-1816, 2001.

[3] D. Rowley y H. Steiner, "Discuss". Faraday Soc., 10, p. 198, 1951.

[4] J. Sauer, Angew. Chem., Int. Ed. Engl. 6: 16, 1967.

[5] K. N. Houk et al. "Faraday Trans". J. Am. Soc., 90, p. 1599, 1994.

[6] K. N. Houk et al. Acc. Chem. Res., 28, p. 81, 1995.

[7] O. Wiest et al. J. Phys. Chem. A, 101, p. 8378, 1997.

[8] I. Fleming, Frontier Orbitals and Organic Chemical Reactions. Capítulo 4. New York: Jhon Wiley and Sons, 1976.

[9] B. S. Jursic, Z. Zdravkovski, J. Mol. Struct (Theochem), 90, p. 215-221, 1995.

[10] A. Venturini, J. Joglar, S. Fustero, y J. González, J. Org. Chem., 62, 12, p. 3919-3926, 1997.

[11] R. A. Sustmann simple model for substituent effects in cycloaddition reactions. I 1,3-dipolar cycloadditions, p. 2717-2720.

[12] J. K. Snyder, M. S. Corbett, X. Liu, A. Sanyal, "Cycloadditions of chiral anthracenes: effect of the trifluoromethyl group". Tetrahedron Letters, 44, p. 931, 2003.

[13] J. K. Snyder, A. Sanyal, "Stereoselective DielsAlder Reactions of Chiral Anthracenes", Org. Lett., 2, p. 2527, 2000.

[14] M. J. Frisch, G. W. Trucks, H. B. Schlegel, G. E. Scuseria, M. A. Robb, J. R. Cheeseman,V. G. Zakrzewski, J. A. Montgomery, R. E. Stratmann, J. C. Burant, S. Dapprich, J. M. Millam, A. D. Daniels, K. N. Kudin, M. C. Strain, O. Farkas, J. Tomasi, V. Barone, M. Cossi, R.
Cammi, B. Mennucci, C. Pomelli, C. Adamo, S. Clifford, J. Ochterski, G. A. Petersson, P. Y. Ayala, Q. Cui, K. Morokuma, D. K. Malick, A. D. Rabuck, K. Raghavachari, J. B. Foresman, J. Cioslowski, J. V. Ortiz, B. B. Stefanov, G. Liu, A. Liashenko, P. Piskorz, I. Komaromi, R. Gomperts, R. L. Martin, D. J. Fox, T. Keith, M. A. Al-Laham, C. Y. Peng, A. Nanayakkara, C. González, M. Challacombe, P.M. W. Gill, B. G. Johnson, W. Chen, M. W. Wong, J. L. Andres, M. Head

[15] M. Dewar, E. Zoebisch y J. Stewart, “AM1: A New General Purpose Quantum Mechanical Molecular Model”. Amer. Chem. Soc., 107, p. 3902-3909, 1985.

[16] D. Becke, J. Chem. Phys. 98: 5648-5652, 1993.

[17] W. J. Hehre, L. Radom, P. Schleyer, J. Pople, $\mathrm{Ab}$ initio Molecular Orbital Theory. New York: Wiley, 1986.

[18] J. K. Snyder, K. Burgess, N. L. Lajkiewicz, A. Sanyal, W. Yan, "A New Chiral Anthracene for the Asymmetric Diels-Alder/Retro-DielsAlder Sequence". Org. Lett., 7, p. 31-34, 2005.

[19] J. K. Snyder, K. Burgess, N. L. Lajkiewicz, A. Sanyal, W. Yan, "A New Chiral Anthracene for the Asymmetric Diels-Alder/Retro-DielsAlder Sequence". Org. Lett., 7, p. 31-34, 2005.

[20] J. K. Snyder, M. S. Corbett, X. Liu, A. Sanyal, "Cycloadditions of chiral anthracenes: effect of the trifluoromethyl group". Tetrahedron Letters, 44, p. 931, 2003.

[21] H. Wang, Y. Wang, K. Li Han, X. Jun Peng, "A DFT Study of Diels-Alder Reactions of oQuinone Methides and Various Substituted Ethenes: Selectivity and Reaction Mechanism". J. Org. Chem. 70, 4911, 2004.

[22] R. Hoffman, "A Claim on the Development of the Frontier Orbital Explanation of Electrocyclic Reactions"; Angew. Chem. Int. Ed.; 43, p. 6586-6590, 2004. 